\title{
Effet Buben, liaison et modèles orthographiques bretons
}

The Buben effect: liaison and Breton orthographic patterns

Jean-Claude Le Ruyet

\section{OpenEdition}

\section{Journals}

Édition électronique

URL : https://journals.openedition.org/lbl/2176

DOl : $10.4000 / \mathrm{lbl} .2176$

ISSN : 2727-9383

Éditeur

Université de Bretagne Occidentale - UBO

\section{Édition imprimée}

Date de publication : 1 novembre 2011

Pagination : 97-109

ISBN : 978-2-901737-92-6

ISSN : $1270-2412$

Référence électronique

Jean-Claude Le Ruyet, "Effet Buben, liaison et modèles orthographiques bretons », La Bretagne Linguistique [En ligne], 16 | 2011, mis en ligne le 01 mai 2021, consulté le 21 mai 2021. URL : http:// journals.openedition.org/lbl/2176; DOl : https://doi.org/10.4000/lbl.2176

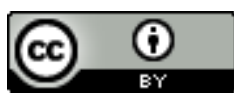

La Bretagne Linguistique est mise à disposition selon les termes de la Licence Creative Commons Attribution 4.0 International. 


\title{
Effet Buben, liaison et modèles orthographiques bretons
}

\begin{abstract}
T,
effet Buben ${ }^{1}$ est l'influence que l'écrit en tant que tel exerce sur la façon dont le lecteur oralise sa langue. J'évoquerai ma tentative de mesurer l'effet Buben dans les filières bilingues actuelles (immersion/parité) et comment on peut envisager l'adaptation orthographique du breton, ces deux points envisagés selon un aspect singulier : celui de la liaison.

Pourquoi la liaison? Curieusement, le sujet intéresse peu en français ${ }^{2}$, et en breton encore moins. Pour le breton, sur une quarantaine d'ouvrages d'enseignement de la langue que j'ai analysés, $52 \%$
\end{abstract}

* Docteur en breton (2009), Rennes 2/ueb

1. L'expression est semble-t-il de Jean-Pierre Chevrot, d'après l'ouvrage de Vladimir Buben, L'influence de l'orthographe sur la prononciation du français contemporain, Bratislava, s. n.,1935.

2. Jules Grévisse y consacre $0.23 \%$ de son monumental Bon usage. John Ågren fut semble-t-il le premier à y consacrer une étude approfondie (1973). Pierre Encrevé a renouvelé l'intérêt pour ce sujet. Mais selon Sandra Caetano, Sandra Santiago et Sofia Alves, «l'un des aspects problématiques de l'apprentissage du français parlé est la liaison, car c'est un phénomène qui a tendance à être peu considéré par les grammairiens et les professeurs» (https://studylibfr.com/ doc/2654707/l-importance-de-la-liaison-dans-la-langue-parlee). 
n'en parlent pas du tout (cf. tableau 1). Seuls quelques-uns mettent le problème à sa juste place. Il s'agirait donc d'abord de rappeler l'existence des liaisons, ensuite de montrer que le système du breton est inverse de celui du français (langue-substrat, ne l'oublions pas, de presque tous les apprenants aujourd'hui, ainsi que de la presque totalité des professeurs) et, troisièmement, de mettre la question de l'écriture de la finale en breton dans une perspective nouvelle (ou renouvelée) : celle de l'effet Buben, que j'ai tenté de cerner.

Tableau 1. Place des liaisons dans les ouvrages d'enseignement du breton

\begin{tabular}{|c|c|c|c|c|c|c|}
\hline $\begin{array}{l}\text { LIAISONS } \\
\text { et livres } \\
\text { d'enseigne- } \\
\text { ment }\end{array}$ & $\begin{array}{l}\text { Aucune } \\
\text { mention }\end{array}$ & $\begin{array}{l}\text { Indiquées uni- } \\
\text { quement dans } \\
\text { les notations } \\
\text { phonétiques }\end{array}$ & $\begin{array}{l}\text { Signalées } \\
\text { par quelques } \\
\text { lignes } \\
\text { seulement }\end{array}$ & $\begin{array}{l}\text { Signalées } \\
\text { (de quelques } \\
\text { lignes à une } \\
\text { page) }\end{array}$ & $\begin{array}{l}\text { Souci pédago- } \\
\text { gique évident } \\
\text { tout le long du } \\
\text { livre }\end{array}$ & Total \\
\hline $\begin{array}{l}\text { Nombre } \\
\text { d'ouvrages } \\
\text { analysés }\end{array}$ & 21 & 2 & 5 & 6 & 6 & 40 \\
\hline$\%$ & $52,5 \%$ & $5 \%$ & $12,5 \%$ & $15 \%$ & $15 \%$ & $100 \%$ \\
\hline
\end{tabular}

J'ai inclus la question des liaisons dans un corpus de quatre règles de prononciation. Ces règles sont bien sûr connues mais la paternité de la formulation de ce corpus revient à Albert Boché qui, à ma connaissance, l'a précisé (oralement) vers le milieu des années 1990. Ces quatre règles pourraient, si elles étaient adoptées et enseignées systématiquement, constituer un cadre dont le but est de conserver au breton, en contact étroit avec le substrat français, ces caractéristiques particulières que sont :

Règle $1: 1$ 'accent tonique.

Règle 2 : la longueur de la voyelle sous l'accent.

Règle 3 : l'assourdissement des consonnes en finale absolue.

Règle 4 enfin : les liaisons.

Pour revenir aux liaisons, j'ai mis en évidence l'influence de l'orthographe sur leur réalisation dans les filières bilingues, du CE2 au lycée, et même chez les adultes-apprenants. Cela à partir de fiches doubles, sur la première les items s'achevant tous par des consonnes douces, sur la seconde par des consonnes dures. Les consonnes sur lesquelles j'ai travaillé sont essentiellement les consonnes corrélatives, ensemble constitué des paires suivantes; $\mathbf{p} / \mathbf{b}, \mathbf{t} / \mathbf{d}, \mathbf{k} / \mathbf{g}, \mathbf{s} / \mathbf{z}$, 
$\mathbf{c h} / \mathbf{j}, \mathbf{f} / \mathbf{v}$. Je ne parlerai ici que des jonctions où l'une ou l'autre de ces consonnes corrélatives se trouve en contact avec une voyelle, une liquide ou une semi-consonne (w, y), laissant délibérément de côté le traitement de $\mathbf{h}$ et $\mathbf{c}^{\mathbf{h}} \mathbf{h}$.

Exemples :

Fiches A1/A2 : liaison devant voyelles (ex : evit $\ulcorner\boldsymbol{d}$ an dud; deus $\curlyvee \boldsymbol{z}$ amañ ; prop $(b$ eo).

Fiches B1/B2 : liaison devant $\mathbf{l}, \mathbf{m}, \mathbf{n}, \mathbf{r}(\mathrm{ex}: \operatorname{Konk} \curlyvee \boldsymbol{g}$-Leon; tok $\ulcorner$ ruz; chañch $\curlyvee j$ roched).

Les graphiques ci-dessous donnent une image de la situation dans les écoles :

Graphique 1. Fiche A2. Total élèves.

Réalisation des liaisons devant voyelle quand la consonne finale est sourde. Comparaison Immersion (Diwan) et Parité horaire (Public et privé catholique)

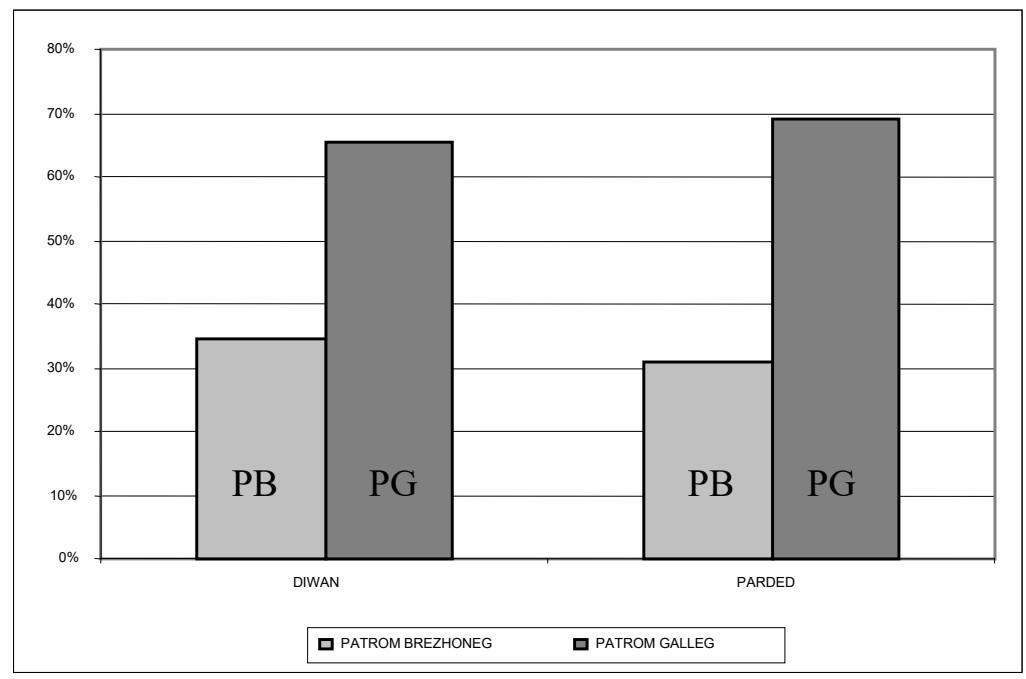

$\mathrm{PB}=$ modèle breton

$\mathrm{PG}=$ modèle français 
Graphique 2. Fiches A1/A2. Total élèves.

Réalisation des liaisons devant voyelle en fonction de la consonne finale. Fiche A1 : consonnes finales douces. Fiche A2 : consonnes finales dures.

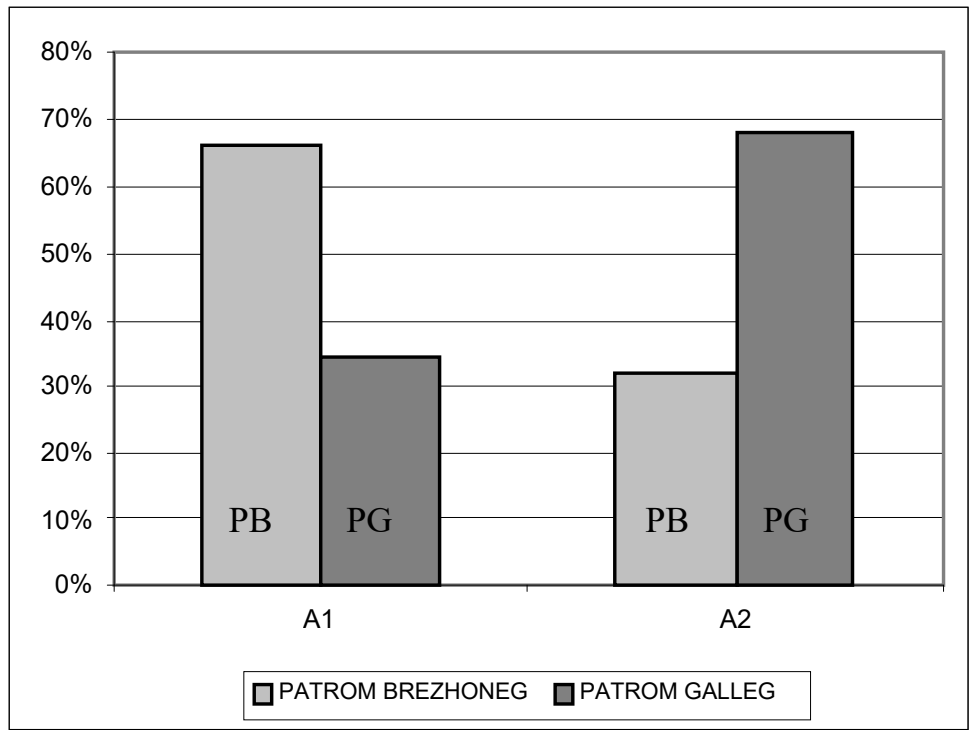

Graphique 3. Fiche B2. Total élèves.

Réalisation des liaisons devant $\mathrm{l}, \mathrm{m}, \mathrm{n}, \mathrm{r}$ quand la consonne finale est sourde. Comparaison Immersion (Diwan) et Parité horaire

(Public et privé catholique).

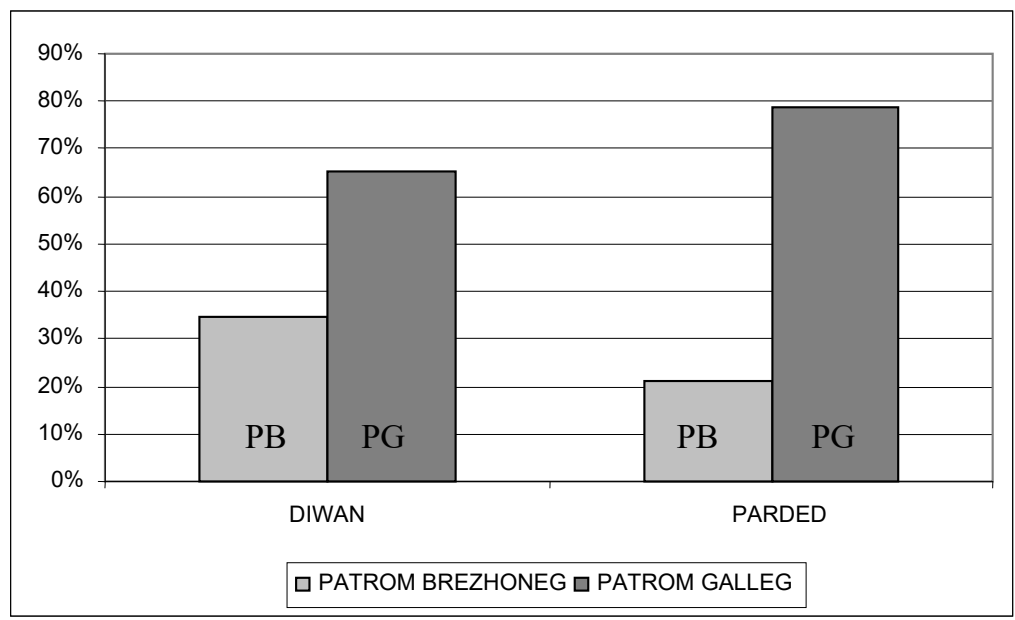


Les résultats, globalement, présentent trois composantes :

1) Devant voyelle notamment (et dans le cas le plus défavorable, c'est-à-dire avec A2, fiche sur laquelle les consonnes finales sont sourdes $>$ graphique 1), il reste un reliquat du système breton des liaisons à hauteur de $30 \%$ environ.

2) L'effet Buben de son côté aurait également une incidence similaire d'un tiers sur la réalisation des liaisons, ce qui est énorme (graphique 2).

3) Cela s'ajoute à l'influence du substrat, non contrecarré par un enseignement volontariste de la question. C'est à ce défaut d'enseignement qu'on peut imputer le dernier tiers. Ainsi donc, de la sorte, environ $60 \%$ des liaisons sont réalisées selon le modèle français, quel que soit le système bilingue porteur, immersion (Diwan) ou parité horaire (filières publiques et catholiques).

Faut-il remédier à cette érosion? Après tout, cela peut être un choix que de laisser le breton s'aligner sur le modèle phonologique $\mathrm{du}$ français, sur son modèle syntaxique et prosodique aussi, cela demande moins d'efforts à tout le monde.

Si l'on opte pour la remédiation, la première chose est d'alerter les enseignants quant au problème dont on parle, alerter aussi les auteurs de manuels, ouvrages pédagogiques, grammaires ${ }^{3}$, car on ne peut plus guère se fier à l'environnement pour corriger les variantes, ou plutôt les déviations par rapport au modèle breton. C'est une révolution que l'on devra opérer, si l'on se réfère à une grammaire récente parue en 2008, qui consacre 18 pages aux mutations $(9 \%$ de l'ouvrage) mais pas un seul mot aux liaisons. Osons parier sur une certaine efficacité de l'école si le problème y est pris à bras-lecorps.

Mais une autre piste s'offre à nous, liée à l'effet Buben, piste qui nous ramène à de vieilles querelles orthographiques. Mais, au lieu de vouloir rallumer de vieux feux inutiles, cette question peut

3. Les ouvrages de TES, maison officielle de diffusion d'ouvrages pédagogiques, dépendante du CRDP, ne sont pas non plus très sensibles à la question. Si la méthode de Mark Kerrain (1997) en parle au fil des pages, la grammaire (collective) de 2009 à destination des collèges et lycées n'en souffle mot. 
être considérée d'un point de vue pragmatique, d'autant qu'elle fait référence au premier point d'accord obtenu par la commission orthographique des années 1971-1976. Or elle concerne directement les liaisons. Il s'agit d'appliquer à tous les mots la règle de la dérivation pour l'écriture de la consonne finale. Peu connaissent l'erreur de Pierre Le Goff et Augustin Guillevic, commise dans leur Grammaire bretonne du dialecte de Vannes (1902), sur les conseils de Joseph Loth, erreur reprise inlassablement ${ }^{4}$ et encore en application dans le système orthographique majoritaire, le seul à être employé dans les écoles bilingues maintenant, depuis la décision compréhensible mais néanmoins arbitraire prise il y a peu par les trois Inspecteurs de l'Education Nationale ayant compétence dans l'enseignement bilingue. Selon ces auteurs vannetais, Pierre Le Goff et Augustin Guillevic, si l'on doit écrire les substantifs en fonction de la dérivation, en revanche toutes "les autres espèces de mots» s'écrivent avec une consonne dure.

À l'époque de Guillevic et Le Goff on ne connaissait pas encore la différence entre les suffixes neutres (la classe la plus nombreuse) et les suffixes durcissants (au nombre de neuf), différence mise en évidence par François Falc'hun cinquante ans plus tard et intégrée par l'Universitaire-55 et par l'Interdialectal- $75^{5}$. Or le principe de la dérivation, appliqué à tous les mots bretons à partir de l'ajout d'un suffixe neutre, ne laisse que peu d'entre eux avec une finale dure. En effet, plus de $85 \%$ des mots bretons se terminent par une douce dans ce cas.

On peut en déduire que les liaisons bretonnes, qui obéissent à la règle de l'adoucissement général devant voyelle, mais aussi devant les liquides $\mathbf{l}, \mathbf{m}, \mathbf{n}, \mathbf{r}$ et devant les semi-voyelles $\mathbf{w}$ et $\mathbf{y}$, seraient facilitées, compte tenu de l'effet Buben, par la présence plus nombreuse sinon générale de consonnes douces en finale.

4. Par Emgleo ar Skrivagnerien (1907), Louis le Clerc (1908), Roparz Hemon (1928, 1942) notamment.

5. J'ai adjoint à chaque orthographe les deux derniers chiffres de leur date de création, ainsi Peurunvan-41 (1941), Skolveureg-55 (1955) et Etrerannyezhel-75 (1975). 
J'ai essayé de déterminer le taux de consonnes douces survenant à la jonction devant les éléments précités dans différents modèles orthographiques existants ou possibles. Plus le taux de consonnes douces est grand, plus il est permis d'attendre de liaisons «à la bretonne» en vertu de l'effet Buben. À l'inverse, bien sûr, plus le taux de consonnes dures est grand, plus grand est aussi le risque d'obtenir des liaisons «à la française». Voici les graphiques dans l'ordre du taux croissant de consonnes douces :

1. Peurunvan-41 (cf. graphique 4).

2. Interdialectal-75 (cf. graphique 5).

3. Synthèse (qui correspond à l'Universitaire-55 pour les finales) (cf. graphique 6).

4. Synthèse $+(=$ Synthèse avec ked au lieu de ket) (cf. graphique 7).

5. Synthèse $++(=$ Synthèse + avec participe passé en - ed $)(\mathrm{cf}$. graphique 8).

Graphique 4. PEURUNVAN-41

Fréquence des consonnes finales en jonction

devant un élément adoucissant

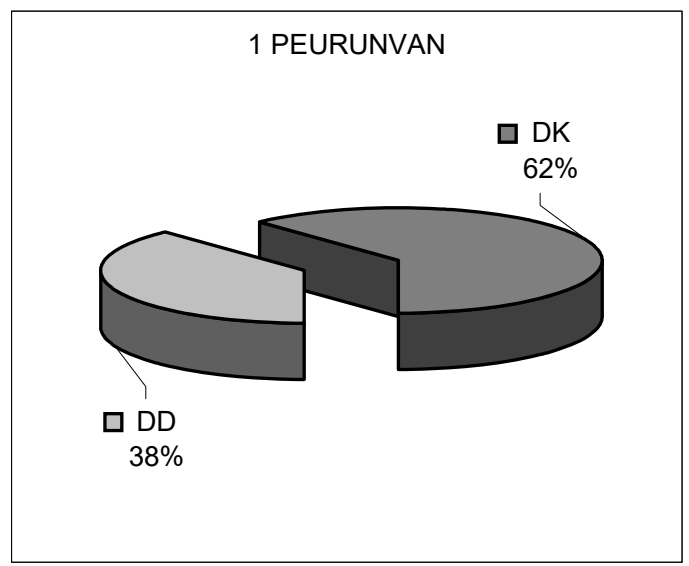

$\mathrm{DD}=$ dibennoù dous, consonnes finales douces

$\mathrm{DK}=$ dibennoù kaled, consonnes finales dures 
Graphique 5. INTERDIALECTAL-75 (avec emploi des deux S) Fréquence des consonnes finales en jonction devant un élément adoucissant. (beaucoup de mots s'écrivent avec-s ou -ss en finale)

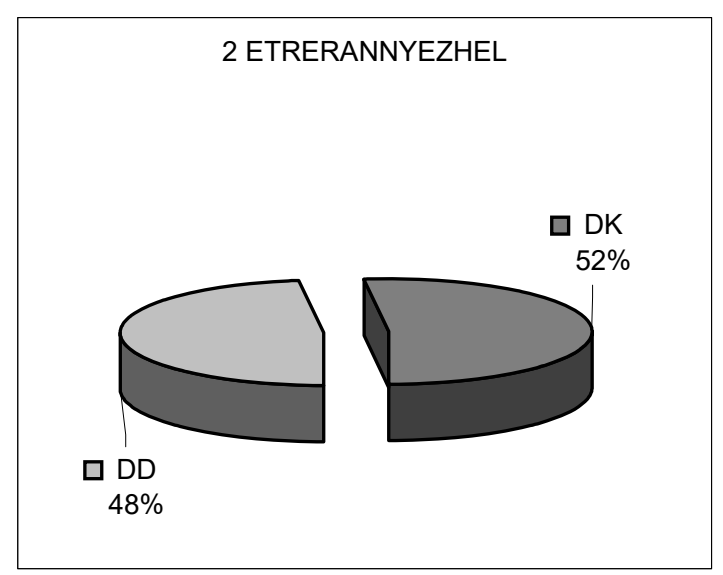

Graphique 6. SYNTHÈSE (= Interdialectal sans les deux S) Fréquence des consonnes finales en jonction devant un élément adoucissant. (ce modèle correspond globalement à celui de l'Universitaire-55 quant à l'écriture des consonnes finales)

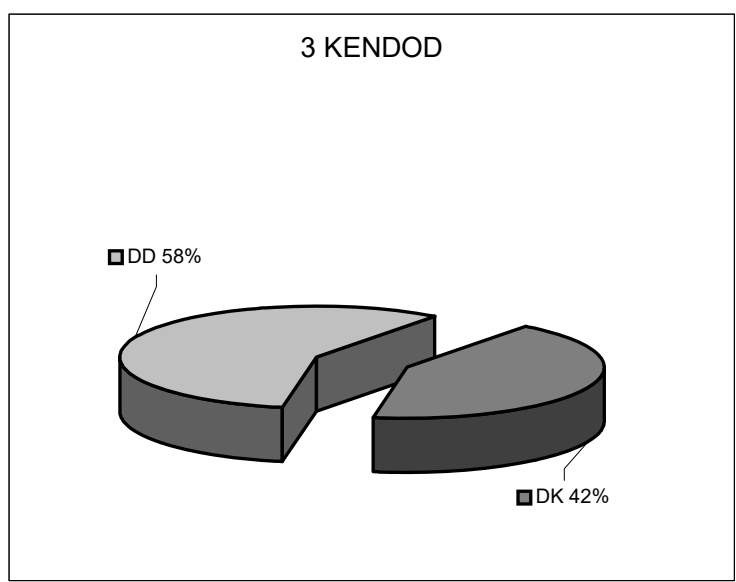


On voit que le système présentant le plus de difficultés est le Peurunvan-41 (fort taux de consonnes dures : seulement 38\% de douces), suivi d'assez près par l'Interdialectal-75 (48\% de douces), en raison de l'adoption des deux $\mathbf{s}$, point qui infère des orthographes comme bras, lous, nos, etc., finales perçues comme dures, mêmes si elles correspondent à un $\mathbf{s}$ doux. Le niveau 3 correspond exactement à l'Universitaire-55 en ce qui concerne les finales. Je l'ai appelé différemment cependant (Synthèse) parce que j'y ai conservé le ZH et le $\mathrm{C}^{\prime} \mathrm{H}$ que l'on trouve à la fois dans le Peurunvan-41 et dans l'Interdialectal-75, traits absents du système Universitaire-55. C'est ce niveau 3 qui obtient le taux maximum de finales douces (58\%), qui est donc le plus facilitant en termes d'apprentissage (quant à la réalisation des liaisons s'entend).

Par curiosité, j'ai ajouté deux autres niveaux, tenant compte de propositions diverses comme l'écriture de $\mathbf{k e d}^{6}$, particule de grande fréquence, pour le niveau 4 (Synthèse + ) ou l'écriture en -ed ${ }^{7} \mathrm{du}$ participe passé pour le niveau 5 (Synthèse ++ ). Voici l'évolution $\mathrm{du}$ taux de consonnes douces en finale en fonction de ces deux extensions (graphique 7 avec ked et graphique 8 avec participe passé en -ed) :

6. L'orthographe ked est en plein accord avec la prononciation de ce mot quand il est accentué (e long), ce qui arrive rarement, mais l'est dans la réponse négative à une question : Braw eo an amzer? Ked ['ke:t].

7. C'est logiquement -ed qu'il faudrait écrire au participe passé (comme en anglais!), si on lui applique également la règle de la dérivation, cf. karedig, kouskedig, dérivés du participe passé où le -d final de kared/kousked se fait bien entendre... 
Graphique 7. SYNTHÈSE + (= Synthèse avec KED au lieu de KET). Fréquence des consonnes finales en jonction devant un élément adoucissant (La fréquence de la particule négative augmente sensiblement à elle seule le taux des consonnes douces DD)

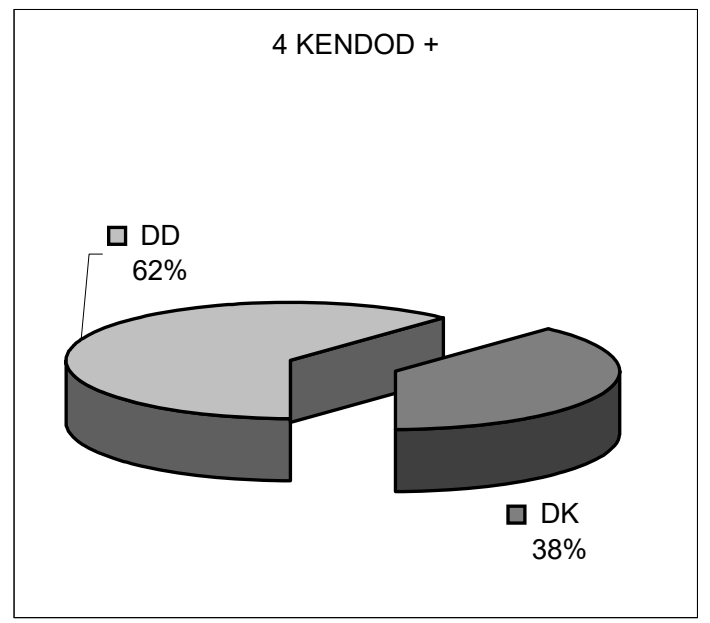

Graphique 8. SYNTHÈSE ++ (= Synthèse + avec participe passé en - ED) Fréquence des consonnes finales en jonction devant un élément adoucissant (Hypothèse basée sur le fait que le participe passé breton est logiquement en -ED, cf. karedig)

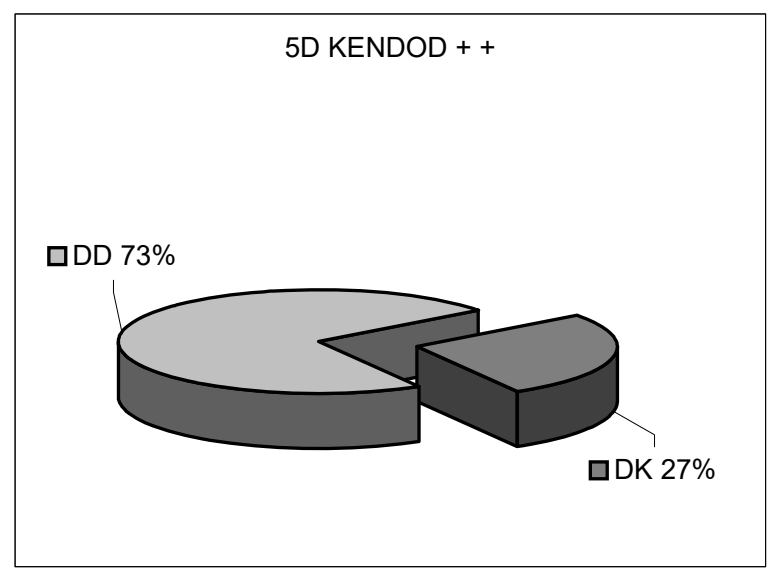


Modifier la consonne finale ne présente pas une grande difficulté lexicographique : il y aurait moins de mots dans les dictionnaires (suppression des doublets comme ${ }^{*}$ bras $^{8}$, *brezhonek, *mut au profit des seuls braz, brezhoneg, mud) et mise en accord de mots comme evid avec la suite evidon, evidout, evidimp, evidoc'h en dépit de eviti, evitañ, evite, modifiés par les suffixes durcissants -(h)i, -(h)añ et -(h)è). Adopter ked ou le participe passé en -ed (comme en anglais) n'est qu'une question de décision.

Je vois trois avantages à cet ajustage de notre outil orthographique :

1) Outre la diminution des entrées, simplification du listing dans le dictionnaire : les familles de mots seraient enfin réunies, sauf naturellement les dérivés construits avec des suffixes durcissants qui resteraient à part (ex : braz, brazadur, brazentez, brazez, brazig... mais brasaad, brasad! brasâdur ${ }^{9}$, brasañ, brasoc'h...

2) Tenant compte de la réalité de l'effet Buben, cette décision faciliterait la réalisation automatique des liaisons dans le sens du système originel breton. Bien qu'insuffisant à lui tout seul, ce dispositif orthographique cohérent, pour peu qu'il soit adjoint à une didactique appropriée, donnerait le maximum de chances au système éducatif pour freiner l'influence du français et, pourquoi pas, inverser la tendance. Entre les différents niveaux présentés, le choix est bien sûr possible en fonction des objectifs envisagés.

3) Enfin, cette mesure qui avait obtenu l'accord général ${ }^{10}$ dans les années 1970, touche à un point capital, puisque le Peurunvan-41 induit des prononciations fausses quant à la longueur de la voyelle

8. Noter cependant le maintien de bras (a bref), pâte à crêpe (W), qui s'opposerait donc à braz (a long). On peut aussi signaler le doublet mad/mat entendu dans la langue. Celle-ci qui joue de l'opposition de longueur pour distinguer l'adjectif de l'adverbe comme ici : mad eo an aval-mañ ['ma :t]/ me 'm eus labouret mat ['mat] (Jean-Yves Plourin, 2000).

9. Il peut être intéressant de signaler par un â (suggestion d'A. Boché) les mots dérivés sur un suffixe durcissant. Dans certains cas d'ailleurs coexistent les deux formes, par exemple à partir de glaz les deux verbes glazañ (-añ suffixe neutre) et glasaad (-(h)aad suffixe durcissant) qui donnent respectivement glazadur verdure et glasâdur, verdissement.

10. Cf. Liste des points d'accord de la commission orthographique, Skol Vreiz, ancienne série, $n^{\circ} 37$, Juillet-Septembre 1974, p. 7. 
dans certains mots, comme les adjectifs monosyllabiques (blot, sot, gwak $^{11}$ ) ou les mots accentués sur la dernière syllabe (fallakr, perak). On pourrait enfin rendre visible à l'écrit la nuance manifestée dans la langue entre des paires telles que mad et mat, la longueur de la voyelle étant pertinente ici : aval-douar mad (pommes de terre bonnes au goût) \# aval-douar mat (pommes de terre de bonne qualité : aspect, taille, conservation, qualités gustatives) (cf. note $\mathrm{n}^{\circ} 9$ ). Cela pourrait aussi permettre de se passer de ces pièges inutiles que présentent certains mots à jonction interne comme *raklavar ou *drouklaouen qui sont à prononcer raglavar et drouglaouen et, évidemment, à écrire de la sorte.

Pour le reste, il ne s'agit plus guère que de points de détail (concernant un certain nombre de mots pris en particulier). Le Peurunvan-41 ainsi relooké présenterait l'avantage d'être l'outil cohérent dont la langue bretonne a besoin au moment où elle commence à devenir visible dans l'espace social. Juste remplacer -41 par un autre chiffre... Serions-nous plus timorés que l'Académie française?

\section{Bibliographie}

ÅGREN John, Étude sur quelques liaisons facultatives dans le français de conversation radiophonique: fréquences et facteurs, UppsalaStockholm, Almqvist och Wiksell, 1973.

BUBEN Vladimir, L'influence de l'orthographe sur la prononciation du français moderne, Bratislava, s. n., 1935.

11. Contrairement à ce que laisse penser l'orthographe, ces trois mots monosyllabiques ont tous une voyelle longue, qui serait facilement détectable si la consonne qui suit était douce, en conformité avec la dérivation normale avec un suffixe neutre : blod/blodenn; sod/sodez; gwag/gwagenn). C'est la même chose pour les mots accentués sur la dernière syllabe : fallagr/fallagriezh; perag/peragiñ). Finis sur ce point les pièges orthographiques («pejoù an doare-skrivañ») déplorés par les auteurs de Yezhadur (TES, 2009, p. 153). 
CHALM Eugène, La grammaire bretonne pour tous, Lannion, An Alarc'h Embannadurioù, 2008.

Chevrot Jean-Pierre, MALderez Isabelle, «L'effet Buben : de la linguistique diachronique à l'approche cognitive (et retour)», Langue Française, vol. 24, n 1, 1999, p. 104-125.

ENCREVÉ Pierre, La liaison avec et sans enchaînement, Paris, Seuil, 1988.

FALC'HUN François, Le système consonantique du breton, avec une étude comparative de phonétique expérimentale, Rennes, Plihon, 1951.

FALC'HUN François, L'orthographe universitaire de la langue bretonne, Brest, Emgleo Breiz, 1956, p. 17-18.

GREVISSE Maurice, Le bon usage, Paris, Gembloux-Duculot, 1986.

Guillevic Augustin \& LE GofF Pierre, Grammaire bretonne du dialecte de Vannes [1901], Brest, Ar skol Vrezoneg, 1986.

HEMON Roparz, Précis de grammaire bretonne, Brest, s. n., 1928.

HEMON Roparz, An doare-skriva nevez, Rennes, s. n., 1942.

KERRAIN Mark, Ni a gomz brezhoneg, Saint-Brieuc, TES, 1997.

LE CleRC Louis, Grammaire bretonne du Dialecte de Tréguier, Prudhomme, Saint-Brieuc [1908], 3e édit., Brest, Ar Skol Vrezoneg Emgleo Breiz, 1986.

LE RUYET Jean-Claude, Komz, liamm ha norm, studiadenn brezantet e stern ur c'horpus peder reolenn-sanañ evid brezhoneg ar skolioù, Thèse de doctorat, Université Rennes 2, 2009.

MorvanNoU Fañch, Initiation au breton sans peine, s. 1., Assimil, 1979.

PlouRIN Jean-Yves, Initiation au breton familier et argotique, Crozon, éditions Armeline, 2000, p. 12-13.

TES (Ti Embann ar Skolioù), Yezhadur, Saint-Brieuc, TES, 2009. 
